\title{
Correlating Radio Astronomy Signals with Many-Core Hardware
}

\author{
Rob V. van Nieuwpoort • John W. Romein
}

Received: 25 September 2009 / Accepted: 11 June 2010 / Published online: 26 June 2010

C The Author(s) 2010. This article is published with open access at Springerlink.com

\begin{abstract}
A recent development in radio astronomy is to replace traditional dishes with many small antennas. The signals are combined to form one large, virtual telescope. The enormous data streams are cross-correlated to filter out noise. This is especially challenging, since the computational demands grow quadratically with the number of data streams. Moreover, the correlator is not only computationally intensive, but also very I/O intensive. The LOFAR telescope, for instance, will produce over 100 terabytes per day. The future SKA telescope will even require in the order of exaflops, and petabits/s of I/O. A recent trend is to correlate in software instead of dedicated hardware, to increase flexibility and to reduce development efforts. We evaluate the correlator algorithm on multi-core CPUs and many-core architectures, such as NVIDIA and ATI GPUs, and the Cell/B.E. The correlator is a streaming, realtime application, and is much more I/O intensive than applications that are typically implemented on many-core hardware today. We compare with the LOFAR production correlator on an IBM Blue Gene/P supercomputer. We investigate performance, power efficiency, and programmability. We identify several important architectural problems which cause architectures to perform suboptimally. Our findings are applicable to data-intensive applications in general. The processing power and memory bandwidth of current GPUs are highly imbalanced for correlation purposes. While the production correlator on the Blue Gene/P achieves a superb $96 \%$ of the theoretical peak performance, this is only $16 \%$ on ATI GPUs, and $32 \%$ on NVIDIA GPUs. The Cell/B.E. processor, in contrast, achieves an excellent $92 \%$. We found that the Cell/B.E. and
\end{abstract}

\footnotetext{
R. V. van Nieuwpoort $(\varangle) \cdot$ J. W. Romein

ASTRON, Netherlands Institute for Radio Astronomy, Oude Hoogeveensedijk 4, 7991 PD Dwingeloo, The Netherlands

e-mail: nieuwpoort@astron.nl

J. W. Romein

e-mail: romein@astron.nl
} 
NVIDIA GPUs are the most energy-efficient solutions, they run the correlator at least 4 times more energy efficiently than the Blue Gene/P. The research presented is an important pathfinder for next-generation telescopes.

Keywords LOFAR · Correlator · Many-core · GPU · Cell/B.E.

CR Subject Classification D.1.3: Parallel programming · J.2: Astronomy

\section{Introduction}

A recent development in radio astronomy is to build instruments where traditional dishes are replaced with many small and simple omni-directional antennas. The signals of the antennas are combined to form one large virtual telescope. Examples include current and future instruments such as LOFAR (LOw Frequency Array) [14], MeerKAT (Karoo Array Telescope) [19], ASKAP (Australian Square Kilometre Array Pathfinder) [8], and SKA (Square Kilometre Array) [16]. These new generation telescopes produce enormous data streams. The data streams from the different antennas must be cross-correlated to filter out noise. The correlation process also performs a data reduction by integrating samples over time. The correlation step is especially challenging, since the computational demands grow quadratically with the number of data streams. The correlator is extremely demanding, since it is not only computationally intensive, but also very data intensive. In the current field of radio astronomy, the number of operations that has to be performed per byte of $\mathrm{I} / \mathrm{O}$ is exceptionally small. For astronomy, high-performance computing is of key importance. Instruments like LOFAR are essentially software telescopes, requiring massive amounts of compute power and data transport capabilities. Future instruments, like the SKA [16], need in the order of exaflops of computation, and petabits/s of I/O.

Traditionally, the online processing for radio-astronomy instruments is done on special-purpose hardware. A relatively recent development is the use of supercomputers $[14,15]$. Both approaches have several important disadvantages. Special-purpose hardware is expensive to design and manufacture and, equally important, it is inflexible. Furthermore, the process from creating a hardware design and translating that into a working implementation takes a long time. Solutions that use a supercomputer (e.g., a Blue Gene/P in the LOFAR case) are more flexible [14], but are expensive to purchase, and have high maintenance and electrical power cost. Moreover, supercomputers are not always well-balanced for our needs. For instance, most supercomputers feature highly efficient double-precision operations, while single precision is sufficient for our applications.

In this paper, we investigate the correlation algorithm on many-core hardware, such as graphics processors (GPUs) [13] and the Cell/B.E. [5]. In contrast to many others, we do not only use NVIDIA GPUs, but also include ATI hardware. In addition, we compare with the LOFAR production implementation on a Blue Gene/P supercomputer [14]. As a reference, we also include multi-core general-purpose processors. There are many advantages to the use of many-core systems: it is a flexible software 
solution, has lower costs in terms of purchase and maintenance, and the power usage is significantly lower than that of a traditional supercomputer.

The correlator differs from applications that were investigated on many-core hardware in the past, because of the correlator's low flop/byte ratio. In addition, it is a streaming real-time application, so host-to-device data transfers are on the critical path. In many other studies, these transfers are not considered.

The production correlator on the Blue Gene/P achieves $96 \%$ of the theoretical peak performance. We demonstrate that the processing power and memory bandwidth of current GPUs are highly imbalanced for correlation purposes. This leads to suboptimal performance. Still, GPUs are considerably more power efficient than the BG/P $(4 \times$ for NVIDIA, and $2.4 \times$ for ATI). The Cell/B.E. processor, in contrast, achieves an excellent $92 \%$ efficiency. The Cell/B.E. runs the correlator 4-6times (depending on the manufacturing process of the Cell/B.E.) more energy efficiently than the Blue Gene/P. The research presented in this paper is an important pathfinder for next-generation telescopes.

The rest of this paper is structured as follows. Section 2 explains how the correlation algorithm works, and why it is important. In Sect. 3, we describe the many-core architectures that we evaluate in detail, finishing with a comparison and discussion. Next, in Sect. 4, we explain how we implemented the correlator algorithm on each of these architectures, and describe the performance we achieve. In Sect. 5, we evaluate, compare, and discuss the results, while identifying the weak and strong points of the architectures. Section 6 discusses related work. In Sect. 7, we investigate if our results and insights can be applied to other applications. Additionally, we discuss scalability issues. Finally, we conclude in Sect. 8.

\section{Correlating Radio Astronomy Signals}

We call a set of receivers that are grouped closely together a station. The data streams from the different stations must be filtered, delays in the signal path must be compensated for, and the data streams from different stations must be cross-correlated. The correlation process performs a data reduction by integrating samples over time. In this paper, we use the LOFAR telescope as an example, but the results apply equally well to other instruments. An overview of the processing needed for the standard imaging pipeline of LOFAR is shown in Fig. 1. The pipeline runs from left to right. The thickness of the lines indicates the size of the data streams. In this paper, we focus on the correlator step (the gray box in Fig. 1), because its costs grow quadratically with the number of stations. All other steps have a lower time complexity. We choose 64 as the number of stations, since that is a realistic number for LOFAR. Future instruments will likely have even more stations. We call the combination of two stations a baseline. The total number of baselines is $(n r$ Stations $\times(n r$ Stations +1$) / 2$, since we need each pair of correlations only once. This includes the autocorrelations (the correlation of a station with itself), since we need this later in the pipeline for calibration purposes. Although the autocorrelations can be computed with fewer instructions, we ignore this here, since the number of autocorrelations is small, and grows linearly with the number of stations, while the number of normal correlations grows quadratically. 


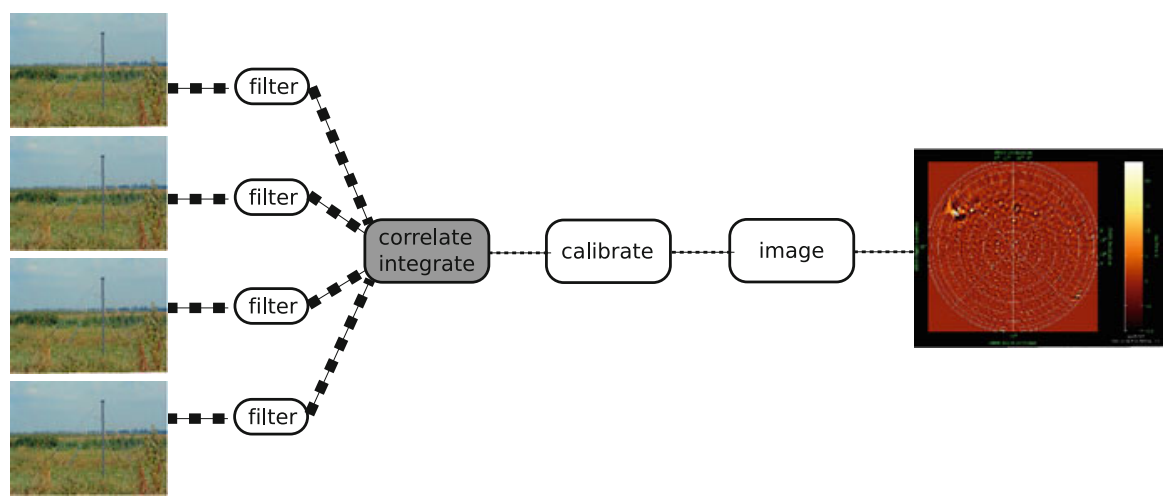

Fig. 1 An extremely simplified view of LOFAR processing

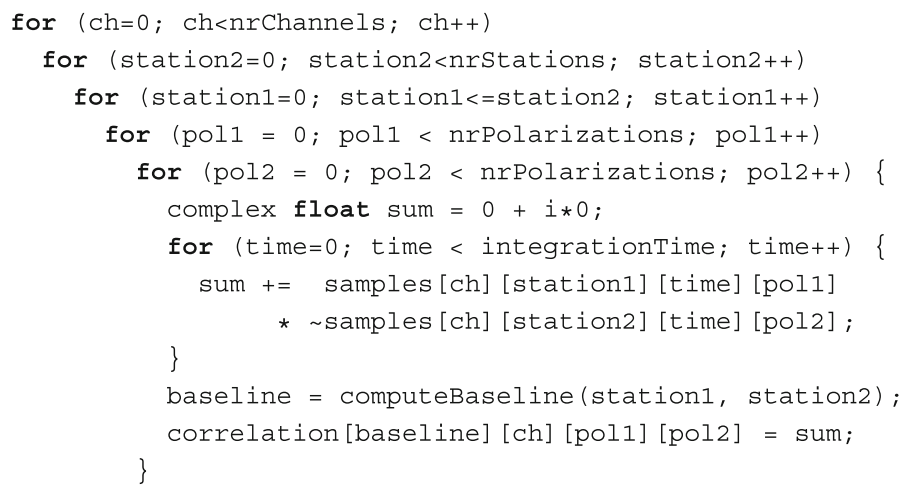

Fig. 2 Pseudo code for the correlation algorithm

The correlator algorithm itself is straightforward, and can be written in a single formula: $C_{s_{1}, s_{2} \geq s_{1}, p_{1} \in\{X, Y\}, p_{2} \in\{X, Y\}}=\sum_{t} Z_{s_{1}, t, p_{1}} * Z_{s_{2}, t, p_{2}}^{*}$

Pseudo code for the algorithm is shown in Fig. 2. A sample is a $(2 \times 32-$ bit $)$ complex number that represents the amplitude and phase of a signal at a particular time. The receivers are polarized; they take separate samples from orthogonal (X and Y) directions. The received signals from sky sources are so weak, that the antennas mainly receive noise. To see if there is statistical coherence in the noise, simultaneous samples of each pair of stations are correlated, by multiplying the sample of one station with the complex conjugate (i.e., the imaginary part is negated) of the sample of the other station. To reduce the output size, the products are integrated, by accumulating all products. For the LOFAR telescope, we accumulate 768 correlations at $763 \mathrm{~Hz}$, so that the integration time is approximately $1 \mathrm{~s}$. This is much shorter than for current telescopes. The short integration time leads to more output data. Since the correlation of station A and B is the complex conjugate of the correlation of station B and A, only one pair is computed. Stations are also autocorrelated, i.e., with themselves. Both polarizations of a station A are correlated with both polarizations of a station 
$\mathrm{B}$, yielding correlations in $\mathrm{XX}, \mathrm{XY}, \mathrm{YX}$, and YY directions. The correlator is mostly multiplying and adding complex numbers.

We can implement the correlation operation very efficiently, with only four fma instructions, doing eight floating-point operations in total. For each pair of stations, we have to do this four times, once for each combination of polarizations. Thus, in total we need 32 operations and load 8 floats ( 32 bytes) from memory, resulting in exactly one FLOP/byte. The number of operations that is performed per byte that has to be loaded from main memory is called the arithmetic intensity [10]. For the correlation algorithm, the arithmetic intensity is extremely low.

An important optimization that we implemented is the reduction of memory loads by the correlator. This is achieved by keeping correlations that are being accumulated in registers, and by reusing samples that are loaded from memory as many times as possible.

A sample can be used multiple times by correlating it with the samples from multiple other stations in the same loop iteration. For example, a sample from station A in the $\mathrm{X}$ polarization that is loaded into a register pair can be correlated with the $\mathrm{X}$ and $\mathrm{Y}$ polarizations of stations B, C and D, using it 6 times. Figure 3 shows how we correlate multiple stations at the same time. Each square represents the XX, XY, YX, and YY correlations of the stations as indicated by row and column number. The figure is triangular, because we compute the correlation of each pair of stations only once. The squares labeled $A$ are autocorrelations, which could be treated specially since they require less computations. The triangle is divided into larger tiles, in this case $2 \times 3$ tiles (the dark gray boxes), but arbitrary sizes are possible. A tile is correlated as a unit. For example, the lower right-hand-side rectangle correlates stations 9 and 10 with stations 0,1 , and 2 .

It is important to tune the tile size to the architecture. We want to make the tile size as large as possible, while still fitting in the register file. This offers the highest level of data reuse. If we have a $w \times h$ tile size, the number of operations is given by flops $=32 \mathrm{wh}$. The number of bytes that has to loaded from memory is $16(w+h)$. The minimum number of registers that is required is $4(1+\min (w, h))+$ $8 w h$. This is the total number of registers, including accumulators, while reusing

Fig. 3 An example correlation triangle

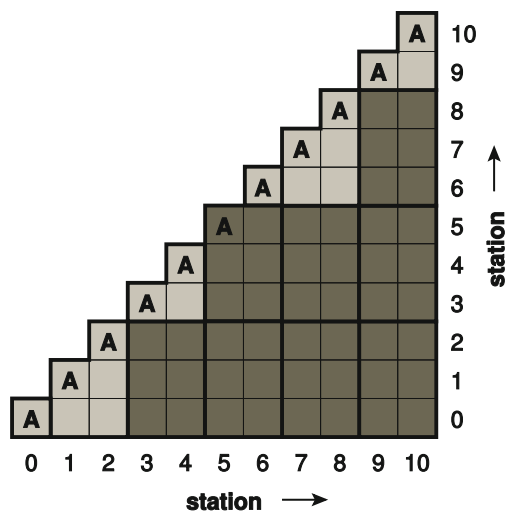


Table 1 Properties of different tile sizes

\begin{tabular}{lllll}
\hline $\begin{array}{l}\text { Tile } \\
\text { size }\end{array}$ & $\begin{array}{l}\text { Floating point } \\
\text { operations }\end{array}$ & $\begin{array}{l}\text { Memory loads } \\
\text { (Bytes) }\end{array}$ & $\begin{array}{l}\text { Arithmetic } \\
\text { intensity }\end{array}$ & $\begin{array}{l}\text { Minimum nr. } \\
\text { registers (floats) }\end{array}$ \\
\hline $1 \times 1$ & 32 & 32 & 1.00 & 16 \\
$1 \times 2$ & 64 & 48 & 1.33 & 24 \\
$1 \times 4$ & 128 & 80 & 1.60 & 40 \\
$2 \times 2$ & 128 & 64 & 2.00 & 44 \\
$3 \times 2$ & 192 & 80 & 2.40 & 60 \\
$3 \times 3$ & 288 & 96 & 3.00 & 88 \\
$4 \times 3$ & 384 & 112 & 3.43 & 112 \\
$4 \times 4$ & 512 & 128 & 4.00 & 148 \\
\hline
\end{tabular}

registers if a value is no longer needed (hence the min operation). However, this formula does not count additional registers that could be needed for data prefetching, address calculations and loop counters. The number of registers is expressed in single-precision float registers. If an architecture has vector registers, the result can be divided by the vector length. Table 1 shows the properties of different tile sizes.

Despite the division of the correlation triangle in tiles, there still is opportunity for additional data reuse between tiles. The tiles within a row or column in the triangle still need the same samples. In addition to registers, caches can thus also be used to increase data reuse. Since we know exactly what data can be reused at what moment, we found it is important to have direct influence on the caches and the thread scheduler. This way, we can make sure that tiles in same row or column are calculated at the same time by different threads. Because the algorithm is extremely data intensive, the resulting optimized implementation on many-cores is typically limited by the architecture's memory bandwidth. The memory aspects of the algorithm are twofold. There is an algorithmic part, the tile size, which is limited by the number of registers. The second aspect is architectural in nature: the cache sizes, cache hierarchy and hit ratio. Together, these two aspects dictate the memory bandwidth that is needed to keep the ALUs busy.

In this paper, we focus on the maximal performance that can be achieved with a single many-core chip. It is important to realize that the correlator itself is trivially parallel, since tens of thousands of frequency channels can be processed independently. This allows us to efficiently exploit many-core hardware. We use floating point instead of integer operations, since all architectures support this well. Single precision floating point is accurate enough for our purposes.

Since the code is relatively straightforward, we implemented the performancecritical kernel in assembly on all architectures. Therefore, this paper really compares the hardware architectures; compilers do not influence the performance. Although we wrote the critical parts in assembly, the additional code was written in the natural programming model for each architecture. Therefore, we also take programmability into account. We do this both for the assembly parts, and the high-level code. The first is a measure of the programmability of the hardware architecture itself. The second gives an indication of the quality of the software stack. 


\section{Many-Core Hardware}

Recent many-core architectures present enormous computational performance at very low costs. For example, the recently introduced ATI $4870 \times 2$ GPU has 1600 compute cores, and achieves a theoretical performance of 2.4 teraflops, all on a single PCI card. This GPU costs less than 500 dollars, resulting in an unprecedented price/performance ratio. The number of cores in the accelerators also increases rapidly. In only a few years, the number of cores has increased from about 16, e.g., in the NVIDIA GeForce 6 series in 2004, to the 1600 cores in the ATI $4870 \times 2$, that became available in 2008. In only 4 years, a 100-fold increase in the number of cores has been realized. During this time, the theoretical peak performance increased from 12 gigaflops to 2.4 teraflops, a growth of a factor 200 .

Reductions in power costs become increasingly important. For LOFAR, for example, a large part of the operational costs is the electrical power consumption. Current supercomputers already focus on power efficiency. The IBM Blue Gene/P, for instance, can perform 384 gflops $/ \mathrm{kW}$ [7], which is significantly better than current general-purpose systems. Nevertheless, modern many-core accelerators are, in theory, even more efficient. For example, an NVIDIA GTX280 GPU has a theoretical peak performance of 3952 gflops/kW, more than ten times better than a Blue Gene/P. The IBM Roadrunner system [2], which is based on the Cell/B.E. processor, became the first super computer to achieve one petaflops. The three most energy efficient supercomputers on the Green500 list, ${ }^{1}$ are similarly based on the Cell/B.E.

Although the number of cores and the theoretical peak performance have increased dramatically, the memory bandwidth did not increase equally fast. In fact, the available memory bandwidth per core is decreasing for many-core architectures at the moment. This introduces significant performance bottlenecks for data intensive applications $[13,17]$. The important question is: How much of the theoretical performance can be reached in practice? In this paper we answer this question for correlation process, we extrapolate our results to data-intensive applications in general. The increase of both the number of cores and the peak performance is much larger for the accelerators than for normal CPUs. This makes many-core accelerators interesting for high-performance computing. In the remainder of this section, we discuss several many-core architectures in detail, and conclude with a summary and discussion of the differences that are essential for the correlator, and for data-intensive applications in general.

\subsection{General Purpose Multi-Core CPU (Intel Core i7 920)}

As a reference, we implemented the correlator on a multi-core general purpose architecture. We use a quad core Intel Core i7 920 CPU (code name Nehalem) at 2.67 GHz. There is $32 \mathrm{~KB}$ of on-chip L1 data cache per core, $256 \mathrm{~KB}$ L2 cache per core, and $8 \mathrm{MB}$ of shared L3 cache. The thermal design power (TDP) is 130 Watts. The theoretical peak performance of the system is 85 gflops, in single precision. The parallelism

\footnotetext{
${ }^{1}$ See http://www.green500.org.
} 
comes from four cores with two-way hyperthreading, and a vector length of four floats, provided by the SSE4 instruction set.

The architecture has several important drawbacks for our application. First, there is no fused multiply-add instruction. Since the correlator performs mostly multiplies and adds, this can cause a performance penalty. The processor does have multiple pipelines, and the multiply and add instructions are executed in different pipelines, allowing eight flops per cycle per core.

Another problem is that SSE's shuffle instructions to move data around in vector registers are more limited than for instance on the Cell/B.E. processor. This complicates an efficient implementation. For the future Intel Larrabee GPU, and for the next generation of Intel processors, both a fused multiply-add instruction and improved shuffle support has been announced.

The number of SSE registers is small (sixteen 128-bit registers), allowing only little data reuse. This is a problem for the correlator, since the tile size is limited by the number of registers. A smaller tile size means less opportunity for data reuse, increasing the memory bandwidth that is required.

\subsection{IBM Blue Gene/P}

The IBM Blue Gene/P (BG/P) [7] is the architecture that is currently used for the LOFAR correlator [14]. Four $850 \mathrm{MHz}$ PowerPC 450 processors are integrated on each Blue Gene/P chip. We found that the BG/P is extremely suitable for our application, since it is highly optimized for processing of complex numbers. The BG/P performs all floating point operations in double precision, which is overkill for our application. The L2 prefetch unit prefetches the sample data efficiently from memory. In contrast to all other architectures we evaluate, the problem is compute bound instead of I/O bound, thanks to the BG/P's high memory bandwidth per operation. It is 3.5-10 times higher than for the other architectures. The ratio between flops and bytes/s of memory bandwidth is exactly 1.0 for the BG/P.

The BG/P has a register file with 32 vector registers of width 2 . Therefore, 64 floating point numbers (with double precision) can be kept in the register file simultaneously. This is the same amount as on the general purpose Intel chip, but an important difference is that the BG/P has 32 registers of width 2, compared to Intel's 16 of width 4. The smaller vector size reduces the amount of shuffle instructions needed. The BG/P is an energy efficient supercomputer. This is accomplished by using many small, lowpower chips, at a low clock frequency. The supercomputer also has excellent I/O capabilities, there are five specialized networks for communication.

\subsection{ATI 4870 GPU (RV 770)}

The most high-end GPU provided by ATI (recently acquired by AMD) is the 4870 [1]. The RV770 processor in the 4870 runs at $750 \mathrm{MHz}$, and has a thermal design power of 160 Watts. The RV770 chip has ten SIMD cores, each containing 16 superscalar streaming processors. Each streaming processor has five independent scalar ALUs. Therefore, the GPU contains $800(10 \times 16 \times 5)$ scalar 32-bit streaming processors. 
The Ultra-Threaded Dispatch Processor controls how the execution units process streams. The theoretical peak performance is 1.2 teraflops. The 4870 has $1 \mathrm{~GB}$ of GDDR5 memory with a theoretical bandwidth of $115.2 \mathrm{~GB} / \mathrm{s}$. The board uses a PCIexpress 2.0 interface for communication with the host system. Each of the ten SIMD cores contains $16 \mathrm{~KB}$ of local memory and separate L1 texture cache. The L2 cache is shared. The maximum L1 bandwidth is $480 \mathrm{~GB} / \mathrm{s}$. The bandwidth between the L1 and L2 Caches is $384 \mathrm{~GB} / \mathrm{s}$. The application can specify if a read should be cached or not. The SIMD cores can exchange data using $16 \mathrm{~KB}$ of global memory.

The ATI 4870 GPU has the largest number of cores of all architectures we evaluate (800). However, the architecture has several important drawbacks for data-intensive applications. First, the host-to-device bandwidth is too low. In practice, the achieved PCI-express bandwidth is far from the theoretical limit. We will explain this in more detail in Sect. 4.3. The achieved bandwidth is not enough to keep all cores busy. Second, we found that overlapping communication with computation by performing asynchronous data transfers between the host and the device has a large impact on kernel performance. We observed kernel slowdowns of a factor of three due to transfers in the background. Third, the architecture does not provide random write access to device memory, but only to host memory. However, for our application which is mostly read-performance bound, this does not have a large impact (see Sect. 4.3).

\subsection{NVIDIA GPU (Tesla C1060)}

NVIDIA's Tesla C1060 contains a GTX 280 GPU (code-named GT200), is manufactured using a $65 \mathrm{~nm}$ process, and has 1.4 billion transistors. The device has 30 cores (called multiprocessors) running at $1296 \mathrm{MHz}$, with 8 single precision ALUs, and one double precision ALU per core. Current NVIDIA GPUs thus have fewer cores than ATI GPUs, but the individual cores are faster. The memory architecture is also quite different. NVIDIA GPUs still use GDDR3 memory, while ATI already uses GDDR5 with the 4870 GPU. The GTX 280 in the Tesla configuration has 4 GB of device memory, and has a thermal design power of 236 Watts. The theoretical peak performance is 933 gflops.

The number of registers is large: there are 16384 32-bit floating point registers per multiprocessor. There also is $16 \mathrm{~KB}$ of shared memory per multiprocessor. This memory is shared between all threads on a multiprocessor, but not globally. There is a total amount of $64 \mathrm{~KB}$ of constant memory on the chip. Finally, texture caching hardware is available. NVIDIA only specifies that "the cache working set for texture memory is between 6 and $8 \mathrm{~KB}$ per multiprocessor" [12]. The application has some control over the caching hardware. It is possible to specify which area of device memory must be cached, while the shared memory is completely managed by the application.

On GPUs, it is possible to synchronize the threads within a multiprocessor. With our application, we exploit this to increase the cache hit ratio. This improves performance considerably on NVIDIA hardware, but not on ATI hardware. When accessing device memory, it is important to make sure that simultaneous memory accesses by different threads are coalesced into a single memory transaction. In contrast to ATI 
hardware, NVIDIA GPUs support random write access to device memory. This allows a programming model that is much closer to traditional models, greatly simplifying software development. The NVIDIA GPUs suffer from a similar problem as the ATI GPUs: the host-to-device bandwidth is equally low.

\subsection{The Cell Broadband Engine (QS21 Blade Server)}

The Cell Broadband Engine (Cell/B.E.) [5] is a heterogeneous many-core processor, designed by Sony, Toshiba and IBM (STI). The Cell/B.E. has nine cores: the Power Processing Element (PPE), acting as a main processor, and eight Synergistic Processing Elements (SPEs) that provide the real processing power. All cores run at 3.2 GHz. The cores, the main memory, and the external I/O are connected by a highbandwidth $(205 \mathrm{~GB} / \mathrm{s})$ Element Interconnection Bus (EIB). The main memory has a high-bandwidth $(25 \mathrm{~GB} / \mathrm{s})$, and uses XDR (Rambus). The PPE's main role is to run the operating system and to coordinate the SPEs. An SPE contains a RISC-core (the Synergistic Processing Unit (SPU)), a $256 \mathrm{~KB}$ Local Store (LS), and a memory flow controller.

The LS is an extremely fast local memory (SRAM) for both code and data and is managed entirely by the application with explicit DMA transfers. The LS can be considered the SPU's L1 cache. The LS bandwidth is $47.7 \mathrm{~GB} / \mathrm{s}$ per SPU. The Cell/B.E. has a large number of registers: each SPU has 128, which are 128-bit (4 floats) wide. The theoretical peak performance of one SPU is 25.6 single-precision gflops. The SPU can dispatch two instructions in each clock cycle using the two pipelines designated even and odd. Most of the arithmetic instructions execute on the even pipe, while most of the memory instructions execute on the odd pipe. We use a QS21 Cell blade with two Cell/B.E. processors and 2 GB main memory (XDR). This is divided into 1 GB per processor. A single Cell/B.E. in our system has a TDP of $70 \mathrm{~W}$. Recently, an equally fast version with a $50 \mathrm{~W}$ TDP has been announced. The 8 SPEs of a single chip in the system have a total theoretical single-precision peak performance of 205 gflops.

\subsection{Hardware Comparison and Discussion}

The memory architectures of the many-core systems are of particular interest, since our application is mostly memory-throughput bound (as will be discussed in Sect. 4). Table 2 shows some key differences of the memory architectures of the many-core systems. Both ATI and NVIDIA GPUs have a hardware L1 and L2 cache, where the application can control which memory area is cached, and which is not. The GPUs also have shared memory, which is completely managed by the application. Also, coalescing and bank conflicts have to be taken into account, at the cost of significant performance penalties [12]. Therefore, the memory access times are nonuniform. The access times of the local store of the Cell/B.E., in contrast, are completely uniform (6 cycles). Also, each Cell/B.E. SPE has its own private local store, there is no cache that is shared between threads. While the GPUs can directly access device memory, the Cell/B.E. does not provide access to main memory. All data has to be 
Table 2 Differences between many-core memory architectures

\begin{tabular}{lll}
\hline Feature & Cell/B.E. & GPUs \\
\hline Access times & Uniform & Non-uniform \\
Cache sharing level & Single thread (SPE) & All threads in a \\
& & multiprocessor \\
Access to off-chip memory & Not possible, only through DMA & Supported \\
Memory access overlapping & Asynchronous DMA & Hardware-managed \\
& & thread preemption \\
Communication & Communication between & Independent thread blocks \\
& SPEs through EIB & + Shared memory within a block \\
\hline
\end{tabular}

Table 3 Properties of the different many-core hardware platforms

\begin{tabular}{llllll}
\hline Architecture & Intel & IBM & ATI & NVIDIA & STI \\
& Core i7 & BG/P & 4870 & Tesla C1060 & Cell/B.E. \\
\hline Cores × FPUs per core & $4 \times 4$ & $4 \times 2$ & $160 \times 5$ & $30 \times 8$ & $8 \times 4$ \\
operations per cycle per FPU & 2 & 2 & 2 & 2 & 2 \\
Clock frequency (GHz) & 2.67 & 0.850 & 0.75 & 1.296 & 3.2 \\
gflops per chip & $\mathbf{8 5}$ & $\mathbf{1 3 . 6}$ & $\mathbf{1 2 0 0}$ & $\mathbf{9 3 6}$ & $\mathbf{2 0 4 . 8}$ \\
Registers per core $\times$ register width & $16 \times 4$ & $64 \times 2$ & $1024 \times 4$ & $2048 \times 1$ & $128 \times 4$ \\
Total L1 data cache size per chip (KB) & 32 & 128 & $? ? ?$ & $? ? ?$ & 2048 \\
Total L1 cache bandwidth (GB/s) & $? ? ?$ & 54.4 & 480 & $? ? ?$ & 409.6 \\
Total device RAM bandwidth (GB/s) & n.a. & n.a. & 115.2 & 102 & n.a. \\
total host RAM bandwidth (GB/s) & $\mathbf{2 5 . 6}$ & $\mathbf{1 3 . 6}$ & $\mathbf{8 . 0}$ & $\mathbf{8 . 0}$ & $\mathbf{2 5 . 8}$ \\
Process Technology (nm) & 45 & 90 & 55 & 65 & 65 \\
TDP (W) & 130 & 24 & 160 & 236 & 70 \\
gflops / Watt (based on TDP) & $\mathbf{0 . 6 5}$ & $\mathbf{0 . 5 7}$ & $\mathbf{7 . 5 0}$ & $\mathbf{3 . 9 7}$ & $\mathbf{2 . 9 3}$ \\
gflops/device bandwidth (gflops / GB/s) & n.a. & n.a. & $\mathbf{1 0 . 4}$ & $\mathbf{9 . 2}$ & n.a. \\
gflops/host bandwidth (gflops / GB/s) & $\mathbf{3 . 3}$ & $\mathbf{1 . 0}$ & $\mathbf{1 5 0}$ & $\mathbf{1 1 7}$ & $\mathbf{7 . 9}$ \\
\hline For the Cel/B.E. & & &
\end{tabular}

For the Cell/B.E., we consider the local store to be L1 cache

Key properties of the architectures are shown in bold

loaded and stored into the local store first. Also, the way that is used to overlap memory accesses with computations is different. The Cell/B.E. uses asynchronous DMA transfers, while the GPUs use hardware-managed thread preemption to hide load delays. Finally, the SPEs of the Cell/B.E. can communicate using the Element Interconnection Bus, while the multiprocessors of a GPU execute completely independently.

Table 3 shows the key properties of the different architectures we discuss here. Note that the performance numbers indicate the theoretical peak. The memory bandwidths of the different architectures show large differences. Due to the PCI-e bus, the host-to-device bandwidth of the GPUs is low. The number of gflops per byte of memory bandwidth gives an indication of the performance of the memory system. 
A lower number means a better balance between memory and compute performance. For the GPUs, we can split this number into a device-to-host component and an internal component. It is clear that the relative performance of the memory system in the Blue Gene/P system is significant higher than that of all the other architectures. The number of gflops that can be achieved per Watt is an indication of the theoretical power efficiency of the hardware. In theory, the many-core architectures are more power efficient than general-purpose systems and the BG/P.

The Bound and Bottleneck analysis $[10,24]$ is a method to gain insight into the performance that can be achieved in practice on a particular platform. Performance is bound both by theoretical peak performance in flops, and the product of the memory bandwidth and the arithmetic intensity $A I$ (the flop/byte ratio): $\operatorname{per} f_{\text {max }}=\min \left(\operatorname{per}_{\text {peak }}, A I \times\right.$ memoryBandwidth $)$. Several important assumptions are made with this method. First, it assumes that the memory bandwidth is independent of the access pattern. Second, it assumes a complete overlap of communication and communication, i.e., all memory latencies are completely hidden. Finally, the method does not take caches into account. Therefore, if the correlator can make effective use of the caching mechanisms, performance can actually be better than $\operatorname{per} f_{\text {max }}$. Nevertheless, the perf $f_{\text {max }}$ gives a rough idea of the performance than can be achieved.

With the GPUs, there are several communication steps that influence the performance. First, the data has to be transferred from the host to the device memory. Next, the data is read from the device memory into registers. Although the GPUs offer high internal memory bandwidths, the host-to-device bandwidth is limited by the low PCIexpress throughput ( $8 \mathrm{~GB} / \mathrm{s}$ for PCI-e $2.016 \mathrm{X}$ ). In practice, we measured even lower throughputs. With the NVIDIA GPU, we achieved $5.58 \mathrm{~GB} / \mathrm{s}$, and with the ATI GPU 4.62 GB/s. For both communication steps, we can compute the arithmetic intensity and the $\operatorname{per} f_{\text {max }}$. The sample data must be loaded into the device memory, but is then reused several times, by the different tiles. We call the arithmetic intensity from the point of view of the entire computation $A I_{\text {global }}$. The number of flops in the computation is the number of baselines times 32 operations, while the number of bytes that have to be loaded in total is 16 bytes times the number of stations. As explained in Sect. 2, the number of baselines is $($ nr Stations $\times(n r$ Stations +1$)) / 2$. If we substitute this, we find that $A I_{\text {global }}=n r$ Stations +1 . Since we use 64 stations, the $A I_{\text {global }}$ is 65 in our case. The $A I_{\text {local }}$ is the arithmetic intensity on the device itself. The value depends on the tile size, and was described in Sect. 2.

For both ATI and NVIDIA hardware, the per $f_{\text {max }}$ global is $65 \times 8.0=520$ gflops, if we use the theoretical PCI-e $2.016 \mathrm{X}$ bandwidth of $8 \mathrm{~GB} / \mathrm{s}$. If we look at the PCI-e bandwidth that is achieved in practice ( 4.62 and $5.58 \mathrm{~GB} / \mathrm{s}$, respectively), the GPUs have a perf $f_{\text {max, global }}$ of only 300 gflops for ATI, and 363 gflops for NVIDIA. Since there is no data reuse between the computations of different frequency channels, this is a realistic upper bound for the performance that can be achieved, assuming there is no performance penalty for overlapping device computation with the host-to-device transfers. We conclude that due to the low PCI-e bandwidth, only a small fraction of the theoretical peak performance can be reached, even if the kernel itself has ideal performance. In the following sections we will evaluate the performance we achieve with the correlator in detail, while comparing to $\operatorname{per} f_{\max }$. 


\section{Correlator Implementation and Performance}

This section describes the implementation of the correlator on the different architectures. In many cases, we experimented with different versions, because it is often unclear beforehand what the best implementation strategy is. We evaluate the performance in detail. For comparison reasons, we use the performance per chip for each architecture. We also calculate the achieved memory bandwidths for all architectures in the same way. We know the number of bytes that has to be loaded by the kernel, depending on the tile size that is used. We divide this by the execution time of the kernel to calculate the bandwidth. Thanks to data reuse with caches and local stores, the achieved bandwidth can be higher than the memory bandwidth.

\subsection{General Purpose Multi-Core CPU (Intel Core i7 920)}

We use the SSE3 instruction set to exploit vector parallelism. Due to the limited shuffle instructions, computing the correlations of the four polarizations within a vector is inefficient. We achieve only a speedup of a factor of 2.8 compared to a version without SSE3. We found that, unlike on all other platforms, vectorizing the integration time loop works significantly better. This way, we compute four samples with subsequent time stamps in a vector. The use of SSE3 improves the performance by a factor of 3.6 in this case. In addition, we use multiple threads to utilize all four cores. To benefit from hyperthreading, we need twice as many threads as cores (i.e., 8 in our case). Using more threads does not help. Hyperthreading increases performance by $6 \%$. The most efficient version uses a tile size of $2 \times 2$. Larger tile sizes are inefficient due to the small SSE3 register file. We achieve a performance of $48.0 \mathrm{gflops,} 67 \%$ of the peak, while using $73 \%$ of the peak bandwidth.

\subsection{IBM Blue Gene/P}

The LOFAR production correlator is implemented on the Blue Gene/P platform. We use it as the reference for performance comparisons. The (assembly) code hides load and instruction latencies, issues concurrent floating point, integer, and load/store instructions, and uses the L2 prefetch buffers in the most optimal way. We use a cell size of $2 \times 2$, since this offers the highest level of reuse, while still fitting in the register file. The performance we achieve with this version is 13.1 gflops per chip, $96 \%$ of the theoretical peak performance. The problem is compute bound, and not I/O bound, thanks to the high memory bandwidth per flop, as is shown in Table 3. For more information, we refer to [14].

\subsection{ATI 4870 GPU (RV 770)}

ATI offers two separate programming models, at different abstraction levels. The low-level programming model is called the "Compute Abstraction Layer" (CAL). CAL provides communication primitives and an intermediate assembly language, 
allowing fine-tuning of device performance. For high-level programming, ATI adopted Brook, which was originally developed at Stanford [3]. ATI's extended version is called Brook+ [1]. We implemented the correlator both with Brook+ and with CAL.

With both Brook+ and CAL, the programmer has to do the vectorization, unlike with NVIDIA GPUs. CAL provides a feature called swizzling, which is used to select parts of vector registers in arithmetic operations. For example, it is possible to perform operations like mul r0, r1.xxyy, r2. zwzw, which selects the $x$ and $y$ components of $r 1$ twice, and performs a vector multiplication with the $z$ and $w$ components of $r 2$, resulting in the four combinations. We extensively exploit this feature in the correlator, and found that this improves readability of the code significantly. Unlike the other architectures, the ATI GPUs are not well documented. Essential information, such as the number of registers, cache sizes, and memory architecture is missing, making it hard to write optimized code. Although the situation improved recently, the documentation is still inadequate. Moreover, the programming tools are insufficient. The high-level Brook+ model does not achieve acceptable performance for our application. The low-level CAL model does, but it is difficult to use.

Synchronizning the threads within a multiprocessor can increase the cache hit ratio, by ensuring that threads that access the same samples are scheduled at roughly the same time. With NVIDIA hardware, this leads to a considerable performance improvement (see Sect. 4.4). However, although the ATI hardware can synchronize the threads within a multiprocessor, we could not achieve performance increases this way.

The architecture also does not provide random write access to device memory. The kernel output can be written to at most 8 output registers (each 4 floats wide). The hardware stores these to predetermined locations in device memory. When using the output registers, at most 32 floating point values can be stored. This effectively limits the tile size to $2 \times 2$. Random write access to host memory is provided. The correlator reduces the data by a large amount, and the results are never reused by the kernel. Therefore, they can be directly streamed to host memory.

The theoretical operations/byte ratio of the ATI 4870 architecture is 10.4 for device memory (see Table 3). In order to achieve this ratio with our application, a minimal tile size of $10 \times 10$ would be needed. This would require at least 822 registers per thread. This is unfeasible, so we cannot achieve the peak performance. Data sharing between tiles using the hardware caches could improve this situation.

The best performing implementation streams the result data directly to host memory, and uses a tile size of $4 \times 3$, thanks to the large number of registers. The kernel itself achieves 420 gflops, which is $35 \%$ of the theoretical peak performance. The achieved device memory bandwidth is $114 \mathrm{~GB} / \mathrm{s}$, which is $99 \%$ of the theoretical maximum. Thanks to the large tile size, the cache hit ratio is $65 \%$. As is shown in Table 1, the arithmetic intensity with this tile size is 3.43 . Therefore, $\operatorname{per} f_{\max }=\min (1200,3.43$ $\times 115.2)=395$. We achieve significantly more than this, thanks to the texture cache.

If we also take the host-to-device transfers into account, performance becomes much worse. We found that the host-to-device throughput is only $4.62 \mathrm{~GB} / \mathrm{s}$ in practice, although the theoretical PCI-e bus bandwidth is $8 \mathrm{~GB} / \mathrm{s}$. The transfer can be done asynchronously, overlapping the computation with host-to-device communication. However, we discovered that the performance of the compute kernel decreases signif- 
icantly if transfers are performed concurrently. For the $4 \times 3$ case, the compute kernel becomes 2.2 times slower, which can be fully attributed to the decrease of device memory throughput. Due to the low I/O performance, we achieve only 190 gflops, $16 \%$ of the theoretical peak. This is $63 \%$ of the per $f_{\max , \text { global }}$ of 300 gflops that we calculated in Sect. 3.6.

\subsection{NVIDIA GPU (Tesla C1060)}

NVIDIA's programming model is called Cuda [12]. Cuda is relatively high-level, and achieves good performance. However, the programmer still has to think about many details such as memory coalescing, the texture cache, etc. An advantage of NVIDIA hardware and Cuda is that the application does not have to do vectorization. This is thanks to the fact that all cores have their own address generation units. All data parallelism is expressed by using threads.

The correlator uses 128-bit reads to load a complex sample with two polarizations with one instruction. Since random write access to device memory is supported (unlike with the ATI hardware), we can simply store the output correlations to device memory. We use the texture cache to speed-up access to the sample data. We do not use it for the output data, since that is written only once, and never read back by the kernel. With Cuda, threads within a thread block can be synchronized. We exploit this feature to let the threads that access the same samples run in lock step. This way, we pay a small synchronization overhead, but we can increase the cache hit ratio significantly. We found that this optimization improved performance by a factor of 2.0.

We also investigated the use of the per-multiprocessor shared memory as an application-managed cache. Others report good results with this approach [18]. However, we found that, for our application, the use of shared memory only led to performance degradation.

The best performing implementation uses a tile size of $3 \times 2$. The optimal tile size is influenced by the way the available registers are used. The register file is a shared resource. A smaller tile size means less register usage, which allows the use of more concurrent threads, hiding load delays. On NVIDIA hardware, we found that the using a relatively small tile size and many threads increases performance.

The kernel itself, without host-to-device communication achieves 314 gflops, which is $34 \%$ of the theoretical peak performance. The achieved device memory bandwidth is $122 \mathrm{~GB} / \mathrm{s}$, which is $120 \%$ of the theoretical maximum. We can reach more than $100 \%$ because we include data reuse. The performance we get with the correlator is significantly improved thanks to this data reuse, which we achieve by exploiting the texture cache. The advantage is large, because separate bandwidth tests show that the theoretical bandwidth cannot be reached in practice. Even in the most optimal case, only $71 \%(72 \mathrm{~GB} / \mathrm{s})$ of the theoretical maximum can be obtained. The arithmetic intensity with this tile size is 2.4 . We can use this to calculate the maximal performance without communication. perf $f_{\max }=\min (966,2.4 \times 102)=245$ gflops. In practice, the performance is better than that: we achieve $128 \%$ of this, thanks to the efficient texture cache. 
Fig. 4 Transposing the memory layout for better memory coalescing on the GPU

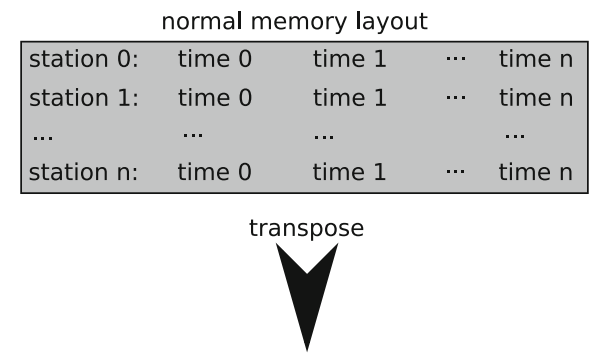

\begin{tabular}{|lllll|}
\multicolumn{5}{c}{ GPU-optimized memory layout } \\
\hline time 0: & station 0 & station 1 & $\cdots$ & station $\mathrm{n}$ \\
time 1: & station 0 & station 1 & $\cdots$ & station $\mathrm{n}$ \\
$\cdots$ & $\cdots$ & $\cdots$ & & $\cdots$ \\
time $\mathrm{n}:$ & station 0 & station 1 & $\cdots$ & station $\mathrm{n}$ \\
\hline
\end{tabular}

If we include communication, the performance drops by $15 \%$, and we only get 274 gflops. Just like with the ATI hardware, this is caused by the low PCI-e bandwidth. With NVIDIA hardware and our data-intensive kernel, we do see significant performance gains by using asynchronous I/O. With synchronous I/O, we achieve only 162 gflops (compared to the 274 we get with asynchronous I/O). Therefore, the use of asynchronous $\mathrm{I} / \mathrm{O}$ is essential.

Since memory coalescing is so important on GPUs, we also investigated an alternative implementation, that first performs a memory transpose. This way, we can make sure that the GPU threads always read the samples in a coalesced way as much as possible. Normally, the memory layout is such that a sequence of samples over time are subsequently stored in memory per station. For the earlier steps in the correlator pipeline, this is the natural order. However, if we change this around, and store the data from all stations for each time step together, we can achieve better performance on the GPU. The two different memory layouts are shown in Fig. 4. Of course, this data transpose is costly. Nevertheless, we found that the total performance in the system can be increased, if we perform the transpose on the host, while the GPU is correlating. Thus, we overlap the computation on the GPU with both the transpose on the host and the data transfer from the host to the device. Performing the transpose on the GPU puts it on the critical path, and decreases performance considerably. Our transpose on the host is implemented using multiple threads to exploit the multi-core architecture, and using SSE instructions for better memory throughput. With this approach, we were able to fully overlap the transpose with useful work on the GPU. Therefore, the transpose is moved completely off the critical path.

A drawback of this approach is that it puts additional stress on the host CPU and memory bus. In a production setup, the host is also used to receive the sample data from the network. Moreover, the CPU could be used to perform additional tasks, such as pre- or post-processing of the data. Possible steps could be filtering, bandpass and phase corrections, etc. It is unclear what would be more efficient: using the host for the data transpose as described above, or using it for additional processing steps. Further research is needed to clarify thus further. 
With the alternative memory layout, the performance of the kernel itself increases from 314 to 357 gflops ( $38 \%$ of the peak). For the version that includes the PCI-e $\mathrm{I} / \mathrm{O}$, the performance increases from 274 to 300 gflops (32\% of the peak). The internal memory bandwidth we achieve is $139 \mathrm{~GB} / \mathrm{s}$. This thus is significantly higher than the $122 \mathrm{~GB} / \mathrm{s}$ we got without the data transpose. In Sect. 3.6, we calculated that the perf $f_{\text {max }}$ global for our hardware is 363 gflops. In practice, we achieve $83 \%$ of this limit due to the external I/O problems.

\subsection{The Cell Broadband Engine (QS21 Blade Server)}

The basic Cell/B.E. programming is based on multi-threading: the PPE spawns threads that execute asynchronously on SPEs. The SPEs can communicate with other SPEs and the PPE, using mechanisms like signals and mailboxes for synchronization and small amounts of data, or DMA transfers for larger data. With the Cell/B.E. it is important to exploit all levels of parallelism. Applications deal with task and data parallelism across multiple SPEs, vector parallelism inside the SPEs, and double or triple-buffering for DMA transfers [5]. The Cell/B.E. can be programmed in $\mathrm{C}$ or $\mathrm{C}++$, while using intrinsics to exploit vector parallelism.

The large number of registers (128 times 4 floats) allows a big tile size of $4 \times 4$, leading to a lot of data reuse. We exploit the vector parallelism of the Cell/B.E. by computing the four polarization combinations in parallel. We found that this performs better than vectorizing over the integration time. This is thanks to the Cell/B.E.'s excellent support for shuffling data around in the vector registers. The shuffle instruction is executed in the odd pipeline, while the arithmetic is executed in the even pipeline, allowing them to overlap.

We identified a minor performance problem with the pipelines of the Cell/B.E. Regrettably, there is no (auto)increment instruction in the odd pipeline. Therefore, loop counters and address calculations have to be performed on the critical path, in the even pipeline. In the time it takes to increment a simple loop counter, four multiplyadds, or 8 flops could have been performed. To circumvent this, we performed loop unrolling in our kernels. This solves the performance problem, but has the unwanted side effect that it uses local store memory, which is better used as data cache.

A distinctive property of the architecture is that cache transfers are explicitly managed by the application, using DMA. This is unlike other architectures, where caches work transparently. Although issuing explicit DMA commands complicates programming, for our application this is not problematic.

By dividing the integration time into smaller intervals, we can keep the sample data for all stations in the local store. We overlap communication with computation, by using multiple buffers. For the sample data we use double buffering. Thanks to the explicit cache, the correlator implementation fetches each sample from main memory only exactly once.

For the correlation output data, several approaches are possible. In [20], we describe an implementation that loads and stores a strip of tiles into the local store with one DMA operation. Consider the example of Fig. 3, for instance, with a tile size of $2 \times 3$. In this case, we load three rows of correlations (the height of a tile) at once, for instance 
the rows 0,1 , and 2. Because of this, we have to load and store the correlations to main memory several times, since the sub-results have to be accumulated. Since the correlations are both read and written, we use triple buffering in this case.

We also developed a version that keeps the correlation results in the local store, and stores the correlation output data to host memory only once. Double buffering is enough in this case. We can fit the result in the local store if the number of receivers is not too large (e.g., 64). For the LOFAR instrument, this is the case. The achieved performance of the two versions is identical. However, both have their own advantages. The first version scales to larger numbers of receivers, but uses more bandwidth between the host memory and the SPEs. This bandwidth is available on the Cell/B.E., so this does not hurt performance. The second version uses less bandwidth, but is limited in the number of receivers. However, the amount of host memory bandwidth used is important, since we may want to run additional operations on the host (the PPE), such as receiving data from the network, and preprocessing it.

If an even larger number of receivers is used (e.g., 256), good performance can still be achieved on the Cell/B.E.. This can be done by splitting the correlation triangle of Fig. 3 in blocks (e.g., of size $32 \times 32$ ), which are in turn divided into the tiles we already used in the previous implementations. On the SPEs, we now only load the samples inside the block into the local store, and not the entire triangle. A complication is that the $\mathrm{X}$ and $\mathrm{Y}$ directions of the blocks no longer deal with the same samples in all cases. For example, in the $\mathrm{X}$ direction, the samples could run from 0 to 31 , while the Y-axis runs from 64 to 96 . Therefore, the number of samples that has to be loaded into the local store increases with a factor of two. Nevertheless, the Cell/B.E. has the bandwidth that is needed for this.

Due to the high memory bandwidth and the ability to reuse data, we achieve 188 gflops, including all memory I/O. This is $92 \%$ of the peak performance on one chip. If we use both chips in the cell blade, the performance drops only with a small amount, and we still achieve $91 \%$ (374 gflops) of the peak performance. Even though the memory bandwidth per operation of the Cell/B.E. is eight times lower than that of the BG/P, we still achieve excellent performance, thanks to the high data reuse factor.

\section{Comparison and Evaluation}

In this section, we compare the performance, power efficiency, and programmablity of the different architectures. We also discuss a relatively new development: OpenCL. We describe how we implemented the correlator using this language.

\subsection{Performance}

Figure 5 shows the performance on all architectures we evaluated. The NVIDIA GPU achieves the highest absolute performance. Nevertheless, the GPU efficiencies are much lower than on the other platforms. The Cell/B.E. achieves the highest efficiency of all many-core architectures, close to that of the BG/P. Although the theoretical peak performance of the Cell/B.E. is 4.6 times lower than the NVIDIA chip, the absolute performance is only 1.6 times lower. If both chips in the QS21 blade are used, the 


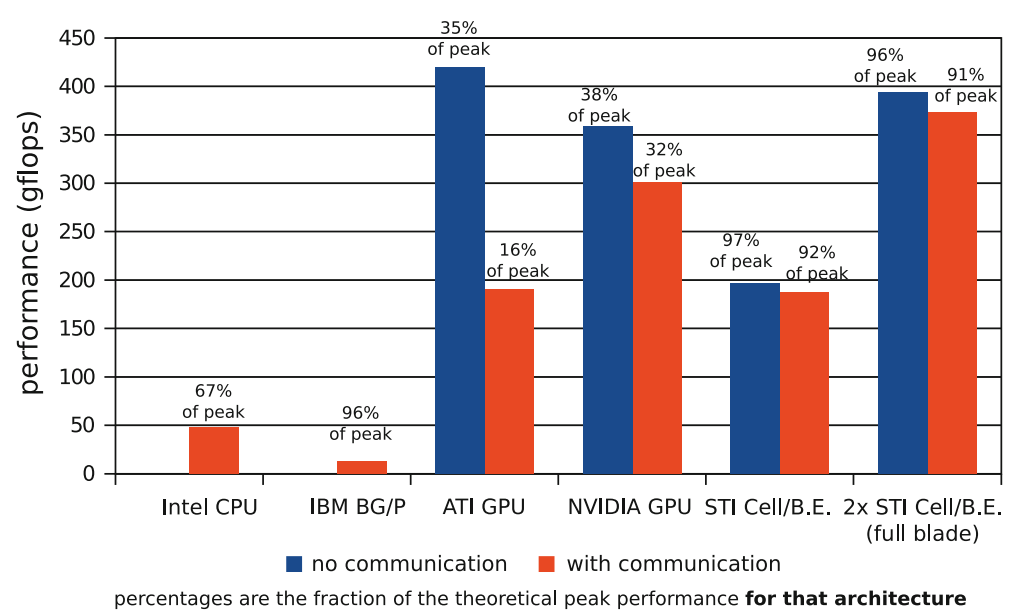

Fig. 5 Achieved performance on the different platforms

Cell/B.E. also has the highest absolute performance. For the GPUs, it is possible to use more than one chip as well. This can be done in the form of multiple PCI-e cards, or with two chips on a single card, as is done with the ATI $4870 \times 2$ device. However, we found that this does not help, since the performance is already limited by the low PCI-e throughput, and the chips have to share this resource. The graph indeed shows that the host-to-device I/O has a large impact on the GPU performance, even when using one chip. With the Cell/B.E., the I/O (from main memory to the Local Store) only has a very small impact.

\subsection{Power Efficiency}

In Table 4, we present the power efficiency for the different architectures. The results show that the Cell/B.E. chip is about five times more energy efficient than the BG/P. This is not a fair comparison, since the BG/P includes a lot of network hardware on chip, while the other architectures do not offer this. Nevertheless, it is clear that the Cell/B.E. is significantly more efficient. A $45 \mathrm{~nm}$ version of the Cell/B.E. has been announced for early 2009. With this version, which has identical performance, but reduces the TDP to about 50W, the Cell/B.E. chip even is seven times more efficient than the BG/P. The $65 \mathrm{~nm}$ version of the Cell/B.E. chip already is about 2.3 times more energy efficient than the GPUs (based on TDP). The fact that the three most energy efficient supercomputers on the Green500 list (see footnote 1) are based on the Cell/B.E. supports our findings. The Green500 list also specifies the achieved power efficiency for entire supercomputers, i.e. including memory, chipsets, networking hardware, etc. PowerXCell-based systems achieve 0.54 glops/W, while the Blue Gene/P is less power efficient, and achieves 0.37 gflops/W. Systems based on general-purpose CPUs only achieve 0.27 gflops/W.

The situation is different if we do not just look at the chips themselves, but also take the full host system into account. The lower three lines in Table 4 show the measured 
Table 4 Measured performance of the different many-core hardware platforms

\begin{tabular}{llllll}
\hline Architecture & $\begin{array}{l}\text { Intel } \\
\text { Core i7 }\end{array}$ & $\begin{array}{l}\text { IBM } \\
\text { BG/P }\end{array}$ & $\begin{array}{l}\text { ATI } \\
4870\end{array}$ & $\begin{array}{l}\text { NVIDIA } \\
\text { Tesla C1060 }\end{array}$ & $\begin{array}{l}\text { STI Cell } \\
\text { (full blade) }\end{array}$ \\
\hline Measured gflops (including I/O) & 48.0 & 13.1 & 190 & 300 & 374 \\
Achieved efficiency & $67 \%$ & $96 \%$ & $16 \%$ & $32 \%$ & $91 \%$ \\
Measured bandwidth (GB/s) & 18.6 & 6.6 & 81 & 139 & 49.5 \\
Bandwidth efficiency & $73 \%$ & $48 \%$ & $70 \%$ & $136 \%$ & $192 \%$ \\
TDP chip (W) & 130 & 24 & 160 & 236 & 140 \\
Theoretical gflops / Watt & 0.65 & 0.57 & 7.50 & 3.97 & 2.93 \\
Achieved gflops/Watt (TDP) & 0.37 & 0.54 & 1.19 & 1.27 & 2.67 \\
Power efficiency compared to BG/P (TDP) & $0.69 \times$ & $1.0 \times$ & $2.20 \times$ & $2.35 \times$ & $4.9 \times$ \\
Measured power, full system (W) & 208 & 44 & 259 & 250 & 315 \\
Achieved gflops/Watt full system & 0.23 & 0.30 & 0.73 & 1.20 & 1.18 \\
Power efficiency compared to BG/P & $0.77 \times$ & $1.0 \times$ & $2.4 \times$ & $4.0 \times$ & $3.9 \times$ \\
\hline
\end{tabular}

power and efficiencies of the full systems, while utilized. For the GPUs, this means that we integrated them in the Core i7 host system that we also use for the general purpose CPU measurements. We measured the dissipated power with a VoltCraft-3000 power meter. The results show that the full Cell blade uses relatively much power in addition to the TDP of the two Cell/B.E. chips. This is partially caused by the XDR memory in the system. As a result of this, the achieved performance per Watt is lower than expected, but still 3.9 times higher than that of the BG/P. For the NVIDIA GPU, however, the opposite is true. In practice, the device consumes much less power than specified by the TDP. Therefore, the achieved efficiency of a full system with the GPU is almost the same as that of the Cell/B.E.. The ATI GPU is much less efficient, due to the small fraction of the theoretical peak performance that is reached in practice.

\subsection{Programmablity}

The performance gap between assembly and a high-level programming language is quite different for the different platforms. It also depends on how much the compiler is helped by manually unrolling loops, eliminating common sub-expressions, the use of register variables, etc., up to a level that the $\mathrm{C}$ code becomes almost as low-level as assembly code. The difference varies between only a few percent to a factor of 10 .

For the BG/P, the performance from compiled $\mathrm{C}++$ code was by far not sufficient. The assembly version hides load and instruction latencies, issues concurrent floating point, integer, and load/store instructions, and uses the L2 prefetch buffers in the most optimal way. The resulting code is approximately 10 times faster than $\mathrm{C}++$ code. For both the Cell/B.E. and the Intel core i7, we found that high-level code in $\mathrm{C}$ or $\mathrm{C}++$ in combination with the use of intrinsics to manually describe the SIMD parallelism yields acceptable performance compared to optimized assembly code. Thus, the programmer specifies which instructions have to be used, but can typically leave the instruction scheduling and register allocation to the compiler. On NVIDIA hardware, 
Table 5 Strengths and weaknesses of the different platforms for data-intensive applications

\begin{tabular}{|c|c|c|c|c|}
\hline $\begin{array}{l}\text { Intel } \\
\text { Core i } 7920\end{array}$ & $\begin{array}{l}\text { IBM } \\
\text { Blue Gene/P }\end{array}$ & $\begin{array}{l}\text { ATI } \\
4870\end{array}$ & $\begin{array}{l}\text { NVIDIA } \\
\text { Tesla C1060 }\end{array}$ & $\begin{array}{l}\text { STI } \\
\text { Cell/B.E. }\end{array}$ \\
\hline +Well-known & $\begin{array}{l}\text { +L2 Prefetch unit } \\
\text { works well } \\
\text { +High memory } \\
\text { bandwidth }\end{array}$ & $\begin{array}{l}\text { +Largest number } \\
\text { of cores } \\
+ \text { Swizzling }\end{array}$ & $\begin{array}{l}\text { + Random } \\
\text { write access } \\
\text { +Cuda is } \\
\text { high-level }\end{array}$ & $\begin{array}{l}\text { +Random } \\
\text { write access } \\
\text { +Shuffle } \\
\text { capabilities } \\
\text { +Explicit cache } \\
\text { +Power efficiency }\end{array}$ \\
\hline $\begin{array}{l}\text {-Few registers } \\
\text {-No fma } \\
\text {-Limited } \\
\text { shuffling }\end{array}$ & $\begin{array}{l}\text {-Everything } \\
\text { double precision } \\
\text {-Expensive }\end{array}$ & $\begin{array}{l}\text { - Low PCI-e } \\
\text { bandwidth } \\
\text { - Transfer slows } \\
\text { down kernel } \\
\text { - No random } \\
\text { write access } \\
\text {-CAL is low-level } \\
\text {-Bad Brook+ } \\
\text { performance } \\
\text { - Not well } \\
\text { documented }\end{array}$ & $\begin{array}{c}\text {-Low PCI-e } \\
\text { bandwidth }\end{array}$ & $\begin{array}{l}\text { - Multiple } \\
\text { parallelism levels } \\
\text { - No increment } \\
\text { in odd pipe }\end{array}$ \\
\hline
\end{tabular}

the high-level Cuda model delivers excellent performance, as long as the programmer helps by using SIMD data types for loads and stores, and separate local variables for values that should be kept in registers. With ATI hardware, this is different. We found that the high-level Brook+ model does not achieve acceptable performance compared to hand-written CAL code. Manually written assembly is more than three times faster. Also, the Brook+ documentation is insufficient.

In Table 5 we summarize the architectural strengths and weaknesses that we identified. Although we focus on the correlator application in this paper, the results are applicable to applications with low flop/byte ratios in general.

\subsection{Implementing the Correlator with OpenCL}

An interesting recent development is the OpenCL programming model [9]. OpenCL is an open standard for parallel programming of heterogeneous systems, developed by the Khronos Group. Many important industry partners participate in the effort. Many-core vendors (e.g., AMD, IBM, Intel, ATI, NVIDIA) have pledged to support OpenCL to increase both portability and programmability of their hardware. The main idea of OpenCL is that a single language is used to program the many-core hardware of all different vendors. We have implemented the correlator application in OpenCL, and tested it with the latest available OpenCL implementations. Currently, AMD provides OpenCL support for multi-core CPUs (not GPUs) in the second beta release of 
their stream SDK version 2.0. NVIDIA released OpenCL support for their GPUs in a closed beta program. Since both implementations are still in the beta stages, we do not discuss performance, but only programmability and portability.

OpenCL consists of two parts: a host API (to interface with the $\mathrm{C}$ program that runs on the CPU), and a special C-based language to develop kernels that run on the accelerators. The host interface is very low-level, and is similar to ATI's CAL, or NVIDIA's driver API. The Cuda model provided by NVIDIA has a much higher abstraction level than OpenCL, and is significantly easier to use. AMD provides an additional set of C++ wrappers around OpenCL's C interface, but this set is not standardized. An important aspect of OpenCL is that it uses run-time compilation for the kernels.

To demonstrate the level of abstraction of the host part of the OpenCL programming model, we will describe the many steps that are necessary to run a kernel. An OpenCL program has to create a context and a command queue. Next, the kernel program has to be loaded from disk (or constructed in memory), and a program object has to be created. Then, the program has to be compiled, and a kernel object has to be created. Subsequently, the arguments to the kernel invocation have to be set, using a call per parameter. Similar to CAL and Cuda, all buffers have to be explicitly allocated and transferred from the host to the device. Finally, a domain has to be specified, indicating the number of global and local threads to use. Only after all these steps, can the kernel be launched. Also, if texture-cached memory is used, as we do with the correlator on GPUs, the programmer has to explicitly create $2 \mathrm{D}$ or $3 \mathrm{D}$ image objects, which also use a different set of calls for the data transfers. Here, the graphics origins of OpenCL clearly shine through.

The language that is used to write the kernels is based on $\mathrm{C}$, with additional extensions. The level of abstraction is similar to Cuda, and of higher level than CAL and the assembly that is needed to program the Cell or CPUs efficiently. A large step forward is that the language has a standard interface to perform vectorization, and also supports swizzling to shuffle data around inside vectors. Special annotations are used to declare data structures in the different memory regions (global, constant, local, and private). Finally, special operations have to be used to load data from texture-cached memory.

We wrote several versions of the correlator in OpenCL, using different tile sizes, and using normal and texture-cached memory. A large benefit of OpenCL is that the code indeed does compile and run without any changes on both CPUs and GPUs. The only exception was a correlator version that uses the texture cache: AMD's beta version does not support this yet. We assume this problem will be solved for the final release.

However, the problem of performance portability is not fully solved by OpenCL yet. First, we had to vectorize the correlator kernel code. With NVIDIA hardware (and the Cuda model), this is not necessary. Therefore, NVIDIA's runtime compiler removes the vectorization. On the CPU, the vectorization did increase performance. Second, the different platforms have very different memory models. The programmer still has to deal with these differences to reach the best performance. The texture cache of the GPUs and the local store of the Cell/B.E. are examples of this. Moreover, we found that our algorithmic changes are still necessary: the different platforms need different 
Table 6 Strengths and weaknesses of openCL

\begin{tabular}{ll}
\hline Strengths & Weaknesses \\
\hline +High-level kernel language & -Low-level host API \\
+Vectorization and swizzling & $\begin{array}{l}\text {-Only C binding standardized } \\
\text { (no C++, Java, or Python) }\end{array}$ \\
+Portability & -Performance portability not solved \\
+Runtime compilation & \\
\hline
\end{tabular}

tile sizes to perform optimally, due to the different numbers of registers they have. Finally, the different architectures require very different numbers of global and local threads: only a few global threads for CPUs, and many thousands, divided over global and local threads, for the GPU. Table 6 summarizes the strengths and weaknesses of OpenCL that we identified.

\section{Related Work}

Intel's 80-core Terascale Processor [11] was the first generally programmable microprocessor to break the teraflop barrier. It has a good flop/Watt ratio, making it an interesting candidate for future correlators.

Intel's Larrabee [17] (to be released) is another promising architecture. Larrabee will be a hybrid between a GPU and a multi-core CPU. It will be compatible with the $\mathrm{x} 86$ architecture, but will have 4-way simultaneous multi-threading, 512-bit wide vector units, shuffle and multiply-add instructions, and special texturing hardware. Larrabee will use in-order execution, and will have coherent caches. Unlike current GPUs, but similar to the Cell/B.E., Larrabee will have a ring bus for communication between cores and for memory transactions.

Another interesting architecture to implement correlators are FPGAs [4]. LOFAR's on-station correlators are also implemented with FPGAs. Solutions with FPGAs combine good performance with flexibility. A disadvantage is that FPGAs are relatively difficult to program efficiently. Also, we want to run more than just the correlator on our hardware. LOFAR is the first of a new generation of software telescopes, and how the processing is done best is still the topic of research, both in astronomy and computer science. We perform the initial processing steps on FPGAs already, but find that this solution is not flexible enough for the rest of the pipeline. For LOFAR, currently twelve different processing pipelines are planned. For example, we would like to do the calibration of the instrument and pulsar detection online on the same hardware, before storing the data to disk. We even need to support multiple different observations simultaneously. All these issues together require enormous flexibility from the processing solution. Therefore, we restrict us to many-cores, and leave applicationspecific instructions and FPGAs as future work. Once the processing pipelines are fully understood, future instruments, such as the SKA, will likely use ASICs.

Williams et al. [23] describe an auto-tuning framework for multi-cores. The framework can automatically perform different low-level optimizations to increase performance. However, GPUs are not considered in this framework. We performed all optimizations manually, which is possible in our case, since the algorithm is relatively 
straightforward. More important, we found that in our case, algorithmic changes are required to achieve good performance. Examples include the use of different tile sizes, and vectorizing over the different polarizations instead of the inner time loop.

A software-managed cache is used on the Cell/B.E. processor. GPUs typically have a small amount of shared memory that can be used in a similar way [18]. An important difference is that in the Cell/B.E. the memory is private for a thread, while with GPUs all threads on a multiprocessor share the memory. The available memory per thread is also much smaller. We applied the technique described in [18], but found it did not increase performance for our application.

Wayth et al. describe a GPU correlator for the Murchison Widefield Array (MWA) [22]. They optimize their code by tiling the correlator triangle in one dimension (a technique described by Harris et.al. [6]), while tiling in two dimensions, as we described in this paper, is much more efficient. For instance, a $2 \times 2$ tile requires the same amount of operations as a $1 \times 4$ tile, but performs fewer memory operations (see Table 1). For larger tiles, the arithmetic intensity of two-dimensional tiles is even better. Also, the MWA GPU version does not use the texture cache, but shared memory. We found that this was significantly slower. Their claim that their GPU implementation is 68 times faster than their CPU implementation is highly biased, since their CPU implementation is not optimized, single threaded, and does not use SSE. As a result, our CPU version is 48 times faster than their CPU version, while our GPU version is 4.2 times faster than their GPU version (even though our data rates are four times as high due to our larger sample sizes). Hence, their GPU implementation is only 1.4 times faster than an optimized CPU implementation, not 68 times.

\section{Discussion}

A key application characteristic of the correlator is that it is extremely regular. This means that we know exactly which memory is referenced at what time. In this paper, we explained that this property makes many optimizations possible. We also implemented several other signal-processing algorithms we did not discuss here, albeit not on all many-core architectures. Most of our conclusions hold for all (data-intensive) applications. However, this paper does not compare the ability of the architectures to cope with unpredictable memory access patterns. We know, for example, that a particular radio-astronomy imaging algorithm (W-projection) exhibits random memory access, and as a result performs poorly on at least some of these architectures, and probably all [21]. Also, the software-manged cache of the Cell/B.E. is less effective here, since the programmer cannot predict the accesses in advance. Fortunately, not all applications behave so unpredictably. In general, we advocate that the focus for optimizations for many-core architectures should be on memory bandwidth, access patterns, and efficient use of the caches, even at the cost of increased synchronization and extra computation.

In this paper, we focus on the maximal performance that can be achieved with a single many-core chip. An exiting result we present here is that even extremely data-intensive applications, such as the correlator, can perform well on many-core architectures, in particular on the Cell/B.E. These results allows us to move forward, 
and bring up the question of scalability: can we scale the results to a full system that processes all telescope data? In this context, it is important to emphasize that the correlator itself is trivially parallel, since tens of thousands of frequency channels can be processed independently. However, in case of an FX correlator, a major data exchange is necessary prior to correlation: each input contains all frequency channels of a single receiver, but the correlator requires a single frequency channel of all receivers. We implemented this for the LOFAR correlator on the 3-D torus of the Blue Gene/P, where we exchange all data asynchronously. Although an efficient implementation is complex, the time required for this exchange is small compared to the time to correlate the data. Moreover, the data rates grow linearly with the number of receivers, while the compute time of the correlator algorithm grows quadratically. We also experimented on a PC cluster with a Myrinet switch, which was able to handle the all-to-all exchange at the required data rates. On the Blue Gene/P, we can scale the application to more than 10,000 cores. For more information, we refer to $[14,15]$.

\section{Conclusions}

Current and future telescopes have high computational and I/O demands. Therefore, we evaluated the performance of the extremely data-intensive correlator algorithm on today's many-core architectures. This research is an important pathfinder for future radio-astronomy instruments. The algorithm is simple, we can therefore afford to optimize and analyze the performance by hand, even if this requires assembly, application-managed caches, etc. The performance of compiler-generated code is thus not an issue: we truly compared the architectural performance.

Compared to the BG/P, many-core architectures have a significantly lower memory bandwidth per operation. Minimizing the number of memory loads per operation is of key importance. We do this by extensively optimizing the algorithm for each architecture. This includes making optimal use of caches and registers. A high memory bandwidth per flop is not strictly necessary, as long as the architecture allows efficient data reuse. This can be achieved through caches, local stores and registers.

Only two architectures perform well with our application. The BG/P supercomputer achieves high efficiencies thanks to the high memory bandwidth per FLOP. The Cell/B.E. also performs excellently, even though its memory bandwidth per operation is eight times lower. We achieve this by exploiting the application-managed cache and the large number of registers, optimally reusing all sample data. The Cell/B.E. is about five to seven times more energy efficient than the BG/P, if we do not take the network hardware into account.

It is clear that application-level control of cache behavior (either through explicit DMA or thread synchronization) has a substantial performance benefit, and is of key importance for data intensive high-performance computing. The results also demonstrated that, for data-intensive applications, the recent trend of increasing the number of cores does not work if $\mathrm{I} / \mathrm{O}$ is not scaled accordingly. 
Acknowledgements This work was performed in the context of the NWO STARE AstroStream project. We gratefully acknowledge NVIDIA, and in particular Dr. David Luebke, for providing freely some of the GPU cards used in this work. Finally, we thank Chris Broekema, Jan David Mol, and Alexander van Amesfoort for their comments on an earlier version of this paper.

Open Access This article is distributed under the terms of the Creative Commons Attribution Noncommercial License which permits any noncommercial use, distribution, and reproduction in any medium, provided the original author(s) and source are credited.

\section{References}

1. Advanced Micro Devices Corporation (AMD): AMD Stream Computing User Guide, Revision 1.1 (2008)

2. Barker, K.J., Davis, K., Hoisie, A., Kerbyson, D.J., Lang, M., Pakin, S., Sancho, J.C.: Entering the petaflop era: the architecture and performance of Roadrunner. In Proceedings of the 2008 ACM/IEEE conference on Supercomputing (SC'08), Austin, Texas. IEEE Press. ISBN:978-1-4244-2835-9 (2008)

3. Buck, I., Foley, T., Horn, D., Sugerman, J., Fatahalian, K., Houston, M., Hanrahan, P.: Brook for GPUs: Stream computing on graphics hardware. In ACM transactions on graphics, Proceedings of SIGGRAPH 2004, pp. 777-786, Los Angeles, California. ACM Press (2004)

4. de Souza, L., Bunton, J.D., Campbell-Wilson, D., Cappallo, R.J. Kincaid, B.: A radio astronomy correlator optimized for the Xilinx Virtex-4 SX FPGA. In international conference on field programmable logic and applications (FPL'07), pp. 62-67, (2007)

5. Gschwind, M., Hofstee, H.P., Flachs, B.K., Hopkins, M., Watanabe, Y., Yamazaki, T.: Synergistic processing in cell's multicore architecture. IEEE Micro. 26(2), 10-24 (2006)

6. Harris, C., Haines, K., Staveley-Smith, L.: GPU accelerated radio astronomy signal convolution. Exp. Astron. 22(1-2), 129-141 (2008)

7. IBMBlue Gene team: Overview of the IBM Blue Gene/P project. IBM J. Res. Develop. 52(1/2), 199-220 (2008)

8. Johnston, S., Taylor, R., Bailes, M. et al.: Science with ASKAP. The Australian square-kilometre-array pathfinder. Exp. Astron. 22(3), 151-273 (2008)

9. Khronos OpenCL Working Group. The opencl specification. version 1.0. See http://www.khronos.org/ opencl/ (2009)

10. Lazowska, E.D., Zahorjana, J., Graham, G.S., Sevcik, K.C.: Quantitative System Performance, Computer System Analysis Using Queueing Network Models. Prentice-Hall, USA (1984)

11. Mattson, T.G., der Wijngaart, R.V., Frumkin, M.: Programming the Intel 80-core network-on-a-chip terascale processor. In Proceedings of the 2008 ACM/IEEE conference on Supercomputing (SC'08), pages 1-11, Austin, Texas, (2008)

12. NVIDIA CUDA Compute Unified Device Architecture Programming Guide Version 2.0, july (2008)

13. Owens, J.D., Luebke, D., Govindaraju, N., Harris, M., Krüger, J., Lefohn, A.E., Purcell, T.: A survey of general-purpose computation on graphics hardware. Comp. Graph. Forum 26(1), 80-113 (2007)

14. Romein, J.W., Broekema, P.C., Mol, J.D., van Nieuwpoort, Rob V.: The LOFAR correlator: implementation and performance analysis. In 15th ACM SIGPLAN Symposium on Principles and Practice of Parallel Programming (PPoPP 2010), Bangalore, India. Accepted for for publication. See http://www. astron.nl/ romein/papers/ (2010)

15. Romein, J.W., Broekema, P.C., van Meijeren, E., van der Schaaf, K., Zwart, W.H.: Astronomical realtime streaming signal processing on a Blue Gene/L supercomputer. In ACM Symposium on Parallel Algorithms and Srchitectures (SPAA'06), pp. 59-66, Cambridge, MA, July (2006)

16. Schilizzi, R.T., Dewdney, P.E.F., Lazio, T.J.W.: The Square Kilometre Array. Proceedings of SPIE, 7012, july (2008)

17. Seiler, L., Carmean, D., Sprangle, E., Forsyth, T., Abrash, M., Dubey, P., Junkins, S., Lake, A., Sugerman, J., Cavin, R., Espasa, R., Grochowski, E., Juan, T., Hanrahan, P.: Larrabee: A many-core x86 architecture for visual computing. ACM Trans. Graph., 27(3), August (2008)

18. Silberstein, M., Schuster, A., Geiger, D., Patney, A., Owens, J.D.: Efficient computation of sumproducts on GPUs through software-managed cache. In Proceedings of the 22nd ACM International Conference on Supercomputing, pp. 309-318, June (2008)

19. The Karoo Array Telescope (MeerKAT). See http://www.ska.ac.za/ 
20. van Nieuwpoort, Rob V., Romein, J.W.: Using many-core hardware to correlate radio astronomy signals. In Proceedings of the ACM International Conference on Supercomputing (ICS'09), pp. 440-449, Yorktown Heights, New York, USA, June (2009)

21. Varbanescu, A., van Amesfoort, A., Cornwell, T., van Diepen, G., van Nieuwpoort, R., Elmegreen, B., Sips, H.: Building high-resolution sky images using the cell/B.E. scientific programming (accepted, to appear) Special issue on high performance computing on the cell BE, (2008)

22. Wayth, R.B., Greenhill, L.J., Briggs, F.H.: A GPU-based real-time software correlation system for the murchison widefield array prototype. Pub. Astron. Soc. Pacific 121, 857-865 (2009)

23. Williams, S., Datta, K., Carter, J., Oliker, L., Half, J., Yelick, K., Bailey, D.: PERI-Auto-tuning memory-intensive kernels for multicore. J. Phys.: Conference Series 125(012038), (2008)

24. Williams, S., Waterman, A., Patterson, D.: Roofline: An insightful visual performance model for floating-point programs and multicore architectures. Communications of the ACM (CACM), (2009). (to appear) 